\title{
Relationships between marbling measures across principal muscles
}

\author{
Małgorzata Konarska ${ }^{a *}$, Keigo Kuchida $^{\mathrm{b}},{\text { Gath } \operatorname{Tarr}^{\mathrm{c}} \text {, Rod Polkinghorne }}^{\mathrm{d}}$
}

${ }^{\text {a }}$ Warsaw University of Life Sciences, Department of Technique and Food Development, Faculty of Human Nutrition and Consumer Sciences, (WULS-SGGW), 159C Nowoursynowska Str., 02-776 Warsaw, Poland

${ }^{\mathrm{b}}$ Obihiro University of Agriculture and Veterinary Medicine, Obihiro 080-8555, Japan

${ }^{c}$ School of Mathematical and Physical Sciences, University of Newcastle, Callaghan, NSW 2308, Australia

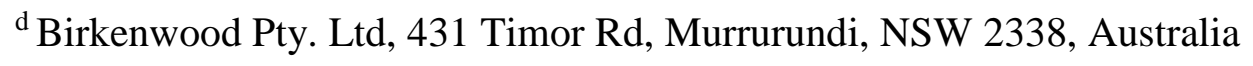

*Corresponding author. Tel.:+48 793756812

E-mail address: malgorzata_konarskal@sggw.pl

\begin{abstract}
As marbling is a principal input into many grading systems it is important to have an accurate and reliable measurement procedure. This paper compares three approaches to measuring marbling: trained personnel, near infrared spectroscopy (NIR) and image analysis. One 25mm slice of meat was utilised from up to 12 cuts from 48 carcasses processed in Poland and France. Each slice was frozen to enable a consistent post slaughter period then thawed for image analysis. The images were appraised by experienced beef graders and the sample used to determine fat content by NIR. We find that image analysis based marbling measures are capturing something different to trained personnel and that there is a strong relationship between near infrared spectroscopy and trained personnel. Finally, we demonstrate that marbling measures taken on one muscle can be predictive of marbling in other muscles in the same carcase. This is particularly important for cut based models such as the Meat Standards Australia system.
\end{abstract}

Keywords: marbling, marbling assessment, beef grading systems, beef carcase classification, correlation analysis, exploratory data analysis

\section{Introduction}

Marbling is an important input into many beef quality grading systems. It is reported to affect taste (Wood et al. 2004; Calkins and Hodgen 2007) and to have a significant relationship to 
consumer acceptance of beef steaks (Platter et al. (2003). If marbling level is to be recognised and encouraged in production systems or utilised as a carcase grading input it needs to be measured accurately, reported to the cattle producer and incorporated within beef classification and payment systems.

In Poland and other European Union (EU) countries beef carcases are evaluated under the EUROP classification system. This system describes carcase conformation (E, U, R, O, P) and external fatness (1-5) but does not report marbling, being focused on describing yield rather than eating quality indicators. The EUROP system is not designed to assess the quality of individual cuts of beef. In Poland, research under the ProOptiBeef project is focused on improving consumer satisfaction with the quality of beef, including consideration of implementing a quality grading system in conjunction with EUROP assessment.

Evaluation of quality grading systems has included both direct collection of data from Polish consumers and beef samples and a review of major existing grading systems, in particular those used in the United States of America (USA), Australia and Japan. In each of these systems, marbling or intramuscular fat (IMF) content is considered one of the main factors to assess beef eating quality as related to consumer satisfaction. (Smith et al. 2013; Anonymous 2014; Anonymous 1996). These classification systems are more complex than the EUROP system and seek to assign levels of consumer satisfaction (eating quality) in addition to yield based measures. The Japan Meat Grading Association (JMGA) system and the United States Department of Agriculture (USDA) system have independent quality and yield grades for a carcase whereas the Australian Meat Standards Australia (MSA) system only describes eating quality, but at a cut rather than carcase level.

The use of image analysis to produce the official JMGA photographic standards and in direct carcase assessment has been reported by Kuchida et al. (2006) and may provide a more sophisticated and objective tool for marbling classification (Kuchida et al. 1997; Kuchida et al. 1998; Kuchida et al. 2000; Kuchida et al. 2001). Konarska et al. (2013) also reported a test procedure for image analysis of beef samples that had been first frozen and then thawed prior to analysis. This procedure was developed to enable collected samples to be assessed under constant laboratory conditions without introducing significant potential bias through extended times between boning at commercial slaughterhouses and image analysis. In brief the procedure applies a constant thaw and bloom time prior to analysis. The current paper incorporates these results in assessing broader issues in relation to alternative measures and intercut relationships. Camera grading of marbling utilizing image analysis of the quartered LD is also allowed under USDA and is becoming more widely used (Moore et al. 2010). 
In Australia the MSA beef grading system is used to predict the quality of beef in relation to consumer acceptance (Ferguson et al. 1999; Polkinghorne et al. 2008; Thompson 2002). The MSA grade is calculated for each cut on the basis of observed or measured carcase traits at grading. Marbling is an important component of the grade calculation and assigned by graders in 10 point increments within a scale of 100 to 1190 supported by 10 digitally produced pictorial standards (Polkinghorne et. al. 2008). In all three systems graders are trained to consider the marbling particle size and distribution in addition to the simple proportion of visual IMF. It is believed that fine, evenly distributed marbling relates to a more consistent and superior eating experience than meat with an equivalent total fat content but irregularly distributed or present in larger coarse particles (Smith 2005). This has important implications in considering potential objective measurement technologies; if total IMF percentage was in fact adequate then non invasive technologies may be able to predict this from a hot or cold carcase scan. If distribution and fineness are important considerations, assessment is far more demanding and likely to require carcase ribbing and evaluation by either visual or photographic image analysis.

In each of the described systems marbling is only assessed on a single cross-section of the M.longissimus thoracis (LT) (JMGA) or M.longissimus thoracis et lumborum (LTL) (USDA and MSA) with this single assessment providing a whole of carcase estimate. In the MSA system marbling effects for individual muscles are defined in relation to the observed LTL marbling site. The marbling effect, related to the LTL observed site, and reported by Watson et al. (2008) varies widely by muscle. What cannot be ascertained however is the extent to which this intermuscle variance relates to a direct effect of marbling in each muscle versus the accuracy of individual cut marbling relationships to LTL marbling. This again has important implications in regard to future grading technologies: if LTL marbling provides similar accuracy to actual individual muscle marbling then a continued focus on accurate measurement of the quartered LTL site is warranted. If not, attention to developing technology that enables individual cut marbling or IMF assessment would rate a higher priority.

The aim of this study was to determine the level of marbling for individual muscles within a range of carcases, to compare alternative marbling measurement approaches and examine correlation between the alternative measures, between individual muscles, and between the LTL assessment site and individual muscles. The study was conducted within the ProOptiBeef project which is evaluating the introduction of a beef quality grading system in Poland. Results of the study will assist in identifying the relative merits of potential marbling 
assessment approaches that could be incorporated in an eating quality assessment system for Polish beef and provides evidence to help inform any improvements to existing international systems.

\section{Methods}

\subsection{Data collection}

Cuts were collected from 27 Polish dairy and dairy by beef (Charolais, Belgian Blue, Limousin) crossbred entire male and female cattle aged between 14 and 24 months grown under the Project "Optimization of beef production in Poland in accordance with the strategy from the fork to the farm" (UDA-POIG.01.03.01-00-204/09-03) after slaughter at a commercial Polish slaughterhouse. Further samples were obtained from 18 female cattle, aged 31 to 178 months, at a French slaughterhouse also including dairy, predominantly Holstein, and beef (Charolais, Croisé, Gascon) breeds. Both slaughterhouses were registered for EU export production and complied with EU regulations for animal welfare, hygiene and further regulations. There were no non standard treatments applied at any point prior to sample fabrication post deboning.

All carcases were graded to EUROP specification prior to chilling. Immediately prior to deboning on the morning after slaughter, carcases were ribbed at the $12 / 13^{\text {th }}$ rib and allowed to bloom for 20 minutes. USDA and MSA grading inputs including marbling, ossification, rib fat depth and $\mathrm{pH}$ were recorded.

Individual primal cuts were collected during deboning and uniquely identified. Approximately 48 hours post slaughter the primal cuts were trimmed of external fat and epimysium and separated into individual muscles. A $25 \mathrm{~mm}$ slice was taken across the grain of each muscle, labelled and vacuum packed.

Twelve muscles were collected in Poland and six in France. Table 1 presents the muscles collected in France and Poland used for image analysis together with the number of each and the associated cut codes, based on MSA description protocols. The codes are a combination of the abbreviation of the common primal cut name and the position of the muscle in the alphabetical list of muscle names (Anonymous, 2005).

The Polish samples were aged 21 days and the French samples for 10 days post slaughter in vacuum packaging at $1{ }^{\circ} \mathrm{C}$. All samples were then frozen and stored at $-20^{\circ} \mathrm{C}$. The frozen samples were transported to the Warsaw University of Life Sciences.

Subsequently they were transferred to a refrigerator 24 hours before photographing and thawed to $2^{\circ} \mathrm{C}$. A test procedure was adopted in which thawed samples were removed from the 
refrigerator, the vacuum packaging removed and the steak then placed on paper towel for 30 minutes to bloom (Konarska et al. 2013). Each sample was then prepared for measurement by drying the surface with paper towel and aligning in a standard position on an A4 sheet of white paper which included a mark to center the steak and the individual sample identification code.

\subsection{Image analysis procedure}

Each sample was photographed by a digital camera system. A NIKON D3200 camera mounted on a jig at $50 \mathrm{~cm}$ height with an APS-C image sensor with a minimum resolution of 15 megapixels and manual settings of ISO 400, shutter speed 1/50 and f-number 3.5. The sample was photographed within a muslim "tent" illuminated by two flash units with a capacity of minimum 200ws. Images were stored as both raw data and reduced to JPEG format. Photographing was carried out on three consecutive days. ColorCheckerPassport (X-rite) was taken for the first shot of each day and used for the colour calibration.

Image analysis was performed using the Beef Analyzer II software (Hayasaka Ricoh Co. Ltd., Sapporo, Japan). To specify muscle area a border line (line width is 1 pixel) was semiautomatically drawn and manually corrected using an image analysis program developed by Kuchida et al. (1997). Further procedures reported by Kuchida et al. (2006) were used to produce a range of marbling measures; the number of marbling particles, two fineness indexes and coarseness indexes of marbling directly and after a thinning procedure removing largest particles. The coarseness index (Ci) was calculated by dividing the area of coarse particles by the total area of all marbling particles. Further coarseness calculations utilised the same procedure after removal of the single largest coarse particle (Ci01), the largest $5(\mathrm{Ci05})$ and the largest 10 (Ci10). The fineness index was defined as the number of small particles per $\mathrm{cm}^{2}$ and the new fineness index calculated by dividing the sum of the circumference of all marbling by the square root of muscle area.

The individual images, saved as JPEG files, were subsequently independently evaluated by highly experienced graders who assigned marbling scores in accordance with the USDA and MSA systems.

\subsection{NIR analysis}

Immediately following photographing each sample was measured using a Minolta CR-400 (Minolta Camera Co., Osaka, Japan) with a measuring aperture diameter of $8 \mathrm{~mm}$ and standard D65 light source ( $2^{\circ}$ standard observer). The colorimeter was calibrated prior to each evaluation session using a reference standard white plate (CR-A43). Five measurements were 
performed for each sample at various points in the sample after 30 minutes at room temperature according to the prescribed method (Tapp et al. 2011). A pH measurement was taken for each sample using a Testo $205 \mathrm{pH}$ meter directly inserted in the sample. The individual cut $\mathrm{pH}$ ranged from 5.06 to 6.42 with a mean of 5.58 and standard deviation 0.17 . Finally, each sample was utilised for near infrared spectrophotometry (NIR) evaluation. A $150 \mathrm{~g}$ sample was homogenised using a Buchi Mixer B-400. The homogenised samples were then placed in a petri dish (90 mm diameter) of the NIR device (near infrared N-solids Flex 500, Buchi, Flawil, Switzerland). For each sample, the scanning spectroscopy was performed three times, in accordance with the methodology of Prevolnik et al. (2004). Software for NIR spectral analysis Ware 1.1 NIR Cal 5.1 (Buchi, Flawil, Switzerland) were used. Moisture, fat, protein, ash and collagen percentages were measured for each sample. Unfortunately 66 samples were lost prior to testing resulting in 294 of the original 360 being analysed.

\subsection{Statistical analysis}

All statistical analyses were performed in R ( $\mathrm{R}$ Core Team, 2015). The primary methods of analysis were Pearson correlation and Spearman correlation estimates. Where missing data was encountered correlations were estimated on a pairwise complete basis, i.e. the set of complete cases were analysed. Principal component analysis was used to identify underlying latent structures in a multivariate data set. The figures were created using the ggplot $2 \mathrm{R}$ package, which also performed some of the analysis, for example, simple linear regressions estimated using ordinary least squares was used to highlight the relationships between pairs of variables and kernel density estimation (Wickham, 2009).

\section{Results and discussion}

\subsection{Carcase measurements}

Data was obtained from 18 French and 27 Polish carcases. Figure 1 summarises the carcase level data and Table 2 provides counts within EUROP and AUS-MEAT categories. In general, the French carcases tended to have higher levels of rib fat, marbling and $\mathrm{pH}$ than the Polish carcases. The French cattle were also older and heavier than the Polish cattle, reflecting typical beef categories in the two countries. Polish carcases tended to have lighter meat colours and exhibited a broader spread of EUROP fatness and conformation scores than French carcases.

It is of interest to determine the strength of any relationships between primary carcase fat and marbling measures. These measures include MSA marbling score, EUROP fat score, rib fat (in 
$\mathrm{mm}$ ) and AUS-MEAT fat colour, presented in Figures 1, 2 and 3. Rib fat and MSA marbling were found to have a moderate Pearson correlation of $0.43(\mathrm{p}<0.01)$. AUS-MEAT fat colour was not significantly correlated with MSA marbling $(p=0.20)$ and rib fat $(p=0.21)$.

The left panel of Figure 3 shows that there is a very weak positive relationship between MSA marbling score and EUROP fat score. If the EUROP fat scores are assumed to be roughly equally spaced and converted to numeric values, e.g. " -2 " is set to 1.7 and " $2+$ " is defined as 2.3 etc., the Pearson correlation between MSA marbling and EUROP fat score is $0.31(\mathrm{p}=0.04)$. This estimate is highly influenced by a couple of outlying observations. In contrast, the Spearman's rank correlation coefficient, which assesses how well the relationship between MSA marbling score and EUROP fat score can be modelled by a monotonic function, is just $0.11(\mathrm{p}=0.48)$.

The right hand side of Figure 3 shows a positive association between EUROP fat score and rib fat depth, for all 45 carcases. The Pearson correlation between EUROP fat score and rib fat depth is $0.31(\mathrm{p}=0.04)$ and the Spearman correlation is $0.34(\mathrm{p}=0.02)$. The relatively low correlation between EUROP fat score and rib fat depth is interesting as both are commonly utilised as indicators of carcase fatness. However, it should be noted that half of the assessed carcases were given a EUROP fat score of 3 and two thirds were either $-3,3$ or $3+$. The difference in variability between rib fat depth and EUROP fat score highlights the crude nature of EUROP fat score and suggests that it would be a very poor predictor of eating quality if it were used in a meat grading model due to the coarse categorisation of carcases.

The results here show that different fat measurements are not highly correlated between carcases. This raises questions in regard to the ability of single point, objectively assessed, fat measures and whole of carcase visual assessments to accurately and reliably describe the fat composition of or distribution within a carcase.

\subsection{MSA marbling, USDA marbling and NIR fat percentage}

The relationships between fat measures of muscle samples are far stronger than the associations between whole of carcase measurements. That is to say, while the inter-carcase fat measures are not strongly correlated, there is good muscle level reliability for certain fat measures.

The 45 carcases yielded 360 individual muscle samples. Each of these 360 samples were individually assessed by independent USDA and MSA assessors and analysed using the various techniques outlined in Section 2. Both assessors were highly experienced, qualified and responsible for data collection within many USA and Australian research trials. Each assessed 
the images independently without consultation. The plots on the main diagonal of Figure 4 are estimated densities and indicate that the distributions of USDA marbling score, MSA marbling score and NIR fat percentages are all right skewed. The scatter plots for the three pairs of variables show a reasonably linear relationship for each pair. Furthermore, there is a strong Pearson correlation $(\mathrm{r}=0.81 ; \mathrm{p}<0.01)$ between direct marbling measures as utilised in the USDA and MSA systems based on 360 observations of individual cut samples by independent expert assessors. Both USDA and MSA marbling were equally well correlated with near infrared spectroscopy (NIR) fat percentage (MSA: $r=0.74, n=293, p<0.01$; USDA: $r=0.74, n$ $=294, \mathrm{p}<0.01)^{1}$. The close agreement between USDA and MSA marbling measures and the similarity of their correlation with NIR fat percentage indicates that either system could be used in carcase or cut assessment with similar effectiveness. The difference in correlation between visual observed marbling and NIR fat percentage however suggests that they are only moderate predictors of each other ( $r$-squared of 0.55 ). This indicates that use of either one alone within carcase grading schemes may result in different rankings and that perhaps an additive approach might be considered.

\subsection{Fat scoring using image analysis}

In contrast to the single point observational marbling and fat measurements the image analysis software output provided a range of measures including a count of all marbling particles; the marbling percentage derived from the relative white versus red area in total; marbling percentage after removal of the single largest particle; as well as a range of coarseness indices of the scanned area and after thinning to identify coarse particles and re-calculated coarseness indexes after removing various percentages of the coarsest particles in addition to the two measures of fineness defined in Section 2.4.

Table 3 presents correlations between the image analysis variables and Figure 5 has densities and scatter plots. Marbling percentage is very strongly correlated with marbling percentage after removal of the single largest particle and both are highly correlated with fineness index and new fineness index. The number of particles is moderately correlated $(\mathrm{p}<0.01)$ with new fineness index $(r=0.64)$ but exhibits negligible correlation with other measures. The various coarseness indices are very highly correlated with each other, but are not at all correlated with the fineness indices. This suggests that they are effective in differentiating fine and coarse fat deposits. Further, the coarseness indices are moderately correlated with the marbling percentage

\footnotetext{
${ }^{1}$ There was one missing observation for MSA marbling score and 66 missing observations for NIR.
} 
and have a marginal negative correlation with the number of particles, reflecting the aggregation of coarse marbling in fewer large deposits.

A principal component analysis was performed to identify any latent structures in the data. All variables were positively correlated with the first principal component (PC) which explained $48 \%$ of the variance. The differences between the variables can be seen by considering the second and third PCs as seen in Figure 6. Together the first three PCs account for 90\% of the variance. There are two clear groups of variables (a) the coarseness indices and (b) MSA marbling, USDA marbling and NIR fat percentage. The remaining variables, number of particles, marbling percentage and the fineness indices, may constitute a third group, though the relationship is less obvious.

To determine the external validity of image analysis techniques, correlations between variables representing observed marbling, NIR fat percentage and image analysis are shown in Table 4. There is negligible correlation between the number of particles and MSA marbling or NIR fat percentage. In contrast, the marbling percentages and the fineness indices are relatively strongly correlated with MSA marbling and NIR fat percentage. The coarseness indices are not strongly correlated with MSA marbling or NIR fat percentage. While the correlation between coarseness index and MSA marbling is statistically different to zero, this is due mostly to the large sample size. The practical importance of such a correlation $(\mathrm{r}=0.16)$ is negligible. The $1 \%$ level of significance was chosen as the significance threshold to minimise the number of false positives given the large number of tests being performed.

The image analysis measures were developed primarily from Japanese Wagyu cattle, which exhibit a high degree of marbling. For our purposes, there is no need to consider four different coarseness indexes, two different fineness indices and two marbling percentage scores. It is possible that the more detailed descriptors within each of the three categories may produce further distinction at very high levels of marbling or on marbling assessed at the $6^{\text {th }} / 7^{\text {th }}$ rib site. For the purposes of this study subsequent cut analysis will focus on MSA marbling score, marbling percentage as determined through image analysis, NIR fat percentage, number of marbling particles, coarseness index $(-5 \%)$ and the new fineness index.

\subsection{Cut level analysis of fat and marbling measures}

Often it is desirable to perform analyses at the cut level, for example, the MSA grading model allows for heterogeneity between cuts. Hence, it is important to understand how different fat measures relate to each other within cuts. That is, which marbling measures are correlated on a cut by cut basis. 
The range and distribution of various marbling measures by cut is illustrated in Figure 7. MSA marbling, NIR fat percentage and new fineness index have similar muscle distributions, for example they identify CHK078 as having a high marbling score and high dispersion and show OYS036 to have a low marbling score with low dispersion.

While it is difficult to draw clear detailed conclusions from Figure 7, some clear cut differences, in particular for OYS036, are evident. While the OYS036 MSA marbling, NIR fat percentage, new fineness index and number of marbling particles have relatively low scores it has very high marbling percentage and coarseness index scores. This reflects the coarse "streaky" marbling pattern in this muscle and may also indicate some difficulty in differentiating marbling from the heavy central sheath of connective tissue. Individual muscles exhibit variation across the measures with BLD096 and RMP131 consistently lower for most.

Figure 8 presents a series of scatter plots of MSA marbling assigned from the individual cut images versus marbling percentage from the image analysis for each of the 12 cuts. The Pearson correlations are given in the top left corner of each scatter plot and can also be found in Table 5 . An approximately linear relationship between MSA marbling and marbling percentage is reasonable for the majority of muscles, however, there are large differences in the range of marbling present and in the slope of the estimated simple linear regression lines. The CHK078 exhibits the largest marbling range although a weaker relationship than the relatively tight linear relationship for KNU099.

More generally, Table 5 presents correlations between MSA marbling assigned from the individual cut images and a range of other marbling measures. While not shown in Table 5, as expected, there is a strong correlation between MSA marbling scores and USDA marbling scores across all cuts. There are also strong relationships between MSA marbling and NIR and marbling percentage. Note that the marbling percentage correlations are the same as those in Figure 8. MSA marbling scores are not correlated with the number of marbling particles or coarseness index, and only significantly correlated with new fineness index for some cuts.

Table 6 presents correlations between NIR fat percentage and image analysis measures. Many cuts show significant correlation between NIR and marbling percentage and new fineness index. Where the relationship is not statistically significant, it is likely due to the low sample sizes. On the other hand, there is no evidence that NIR is correlated with the number of particles or coarseness index.

Table 7 presents correlations between marbling percentage and other image analysis variables. Similarly, to what was found at an aggregate level in Tables 3 and 4, marbling 
percentage is very strongly correlated with new fineness index but only moderately correlated with coarseness and the number of particles.

Across the different muscles, coarseness index was not significantly correlated with new fineness index or number of marbling particles. However, there was a significant correlation between number of particles and new fineness index for all muscles. This is interesting because, the number of particles did not seem to correlate strongly with any of the other variables, however new fineness index did correlate with other variables, especially marbling percentage. This reinforces the conclusions from the aggregate analysis in section 3.3. Further evaluation including relationships to sensory evaluation may provide more explanation of these observations and the related question as to the possible importance of marbling fineness and coarseness relative to overall fat content. A natural extension is to ask whether fat measurements taken on one muscle are representative of other muscles.

\subsection{Intercut marbling relationships}

Further analysis was conducted to address the marbling relationship between muscles within a carcase. This is an important issue for the development of accurate prediction models for beef eating quality and the appropriate weighting of marbling within such a grading system. Marbling at the LTL quartering site is one of several inputs to the MSA model with its contribution to each muscle derived from analysis of consumer responses (Watson et al. 2008). This approach allows for a differentiated muscle effect based on an estimated relationship between observed LTL marbling and the corresponding eating quality of other muscles. A limitation of this approach is accurately determining the relationship between marbling at the LTL quartering site and marbling in other muscles. Hence, the predictive ability of such a model is determined by the extent to which the relationships between actual cut marbling and assessed LTL marbling vary. This section compares marbling measures between individual muscles and the LTL muscle as measured at grading and considers within carcase LTL image analysis in order to provide some guidance as to the strength of the relationship.

To get an understanding of the variability inherent in the marbling scoring process over time, we estimated the Pearson correlation between MSA marbling scores performed at grading, approximately 48 hours post slaughter, and the follow up MSA grading of images for the $44 \mathrm{LL}$ (STR045) cuts $^{2}$. We obtained an estimated correlation of $0.65(\mathrm{p}<0.01)$, that can be seen in Figure 9 and Table 8. This is the tightest possible comparison as the same muscle (LL) was

\footnotetext{
2 One carcase did not have a follow up LD cut available. See Table 1 for a breakdown of the cuts used in the analyses.
} 
observed at initial carcase grading post slaughter and later from image analysis after freezing and thawing, hence representing a gold standard, in the sense that if the correlation between the LL MSA marbling score at initial carcase grading and the follow up image based grading of other muscles approaches or exceeds 0.65 then we can safely assume that measuring only the LL marbling at carcase grading is appropriate to give us an understanding of the marbling present in other muscles. Figure 9 shows a series of scatter plots that illustrate the relationship between MSA LL marbling scores at carcase grading and the later MSA marbling score from image analysis for other cuts. It is clear from the plots that the strength of the dependence varies across muscles, however, with the exception of OUT005 and OYS036, the strength of the relationship is quite strong. To give a specific example, the correlation between MSA marbling score of LL at carcase grading and the subsequent MSA marbling score evaluated using images taken of the CUB045 after freezing and thawing is $0.63(\mathrm{p}<0.01)$.

Correlations between MSA marbling assessment of the LTL cross section at carcase grading and subsequent individual muscle assessments after freezing and thawing are shown in Table 9 with the need to examine this question raised by Konarska et al. (2013). As these correlations may be affected by the time delay and any temperature variation between carcase grading and image analysis together with the freezing and thawing process, correlations between the STR045 image (the closest image to the grading site) and alternative marbling measures for individual cuts was conducted with results shown in Table 9. The general pattern remains the same between the two tables, regardless of whether the MSA marbling score at carcase grading post slaughter is used or the MSA marbling score for the STR045 image assigned from images.

In both Table 8 and 9, there is significant correlation between MSA LTL marbling scores and other muscles. Furthermore, MSA LTL marbling is significantly correlated with NIR fat percentage and somewhat correlated with marbling percentage. These results indicate that it may be reasonable to use striploin marbling at the LTL grading site as a proxy for the majority of larger carcase muscles. An exception is OYS036 which requires further investigation. It is hypothesised that the large central connective tissue sheaf and coarse nature of the marbling particles may create assessment difficulties for both graders and image analysis software. As in the prior intracut analyses, there is negligible correlation between MSA marbling and the number of particles, coarseness index and new fineness index indicating that the image analysis output reflects somewhat different parameters. Given this, the potential to utilise both measures might be considered to improve prediction of sensory outcomes. 
The analysis was replicated after substituting TOP073 and TDR062 for STR045 (results not shown). Correlations between TOP073 and other muscles were very similar to those shown in Table 9 for STR045, however TDR062 exhibited far lower correlations with the other muscles. This raises the possibility of substituting TOP073 or other suitable muscles for STR045 if automated objective measurement approaches such as NIR fat percentage are used or in cases where the carcase is not ribbed.

To summarise, these results show that marbling at the LTL is predictive of marbling in many other muscles, though the strength of the relationship varies between muscles. The proportion of MSA marbling variability in other muscles that can be explained by MSA marbling at the LTL ranges from $24 \%$ for OUT005 (which does not typically exhibit a high degree of marbling) up to $69 \%$ for CUB045 (which is the LT portion of the LTL). Furthermore, there is consistency over time and temperature, in that it does not matter if the marbling scores are taken at slaughter or after the muscle has been frozen and thawed.

\subsection{Potential application in a Polish quality grading system}

This study determined the level of marbling for individual muscles within a range of carcases, compared alternative marbling measurement approaches and examined the correlation between the alternative measures, between individual muscles, and between the LTL assessment site and individual muscles.

At the carcase level, we found that EUROP fat scores were only loosely correlated with rib fat depth and not correlated with MSA marbling score. Furthermore, there was limited variability in EUROP fat score relative to that observed in rib fat depth and MSA marbling scores. Hence, EUROP fat score would not be a useful input into a meat grading model in the sense that it has limited ability to discriminate between carcases. MSA and USDA marbling grading methodologies were closely aligned and both were highly correlated with NIR fat percentage. This raises an interesting question as to whether NIR fat percentage can be used as an objective measure replacement for manual marbling assessment. This will be explored further in a subsequent study which will consider the ability of various marbling measures to predict eating quality.

We also considered a range of image analysis based approaches to measure fat and marbling. We found that all the coarseness indices were highly correlated and recommended proceeding with the trimmed coarseness index that removed 5 of the largest particles as this gives the index a certain level of robustness. The number of particles was found to have moderate correlation with new fineness index but very little correlation with any of the other marbling 
measures. Image based marbling percentage is correlated with new fineness index and the coarseness indices but not the number of particles.

It will be interesting to see which of these measures are predictive of eating quality. This may be central to grading system development, either indicating that prediction is improved by utilising measures of marbling form and distribution or alternatively, that a basic overall fat content measure such as NIR output provides similar accuracy.

The cut level analysis largely mirrored what was found at the aggregate level. There was little variation between the relationships we observed across cuts, except to note that for OYS036, RMP131, CUB045 and BLD096 the correlation between MSA marbling and image assessed marbling percentage was not statistically significant.

Finally, we explored the correlations between marbling measures on cuts between muscles on a given carcase. We found a significant relationship between MSA marbling scores taken at the LTL muscle grading site and other muscles. Furthermore, MSA marbling at LTL is significantly correlated with NIR fat percentage in other muscles with the only exception being OYS036, though this is to be expected, given that we found no significant correlation between OYS036 MSA marbling and OYS036 NIR fat percentage. The results show that marbling of the LTL and TOP073 is predictive of marbling in many other muscles, though the strength of the relationship varies between muscles.

The consistently high correlation between MSA marbling and NIR fat percentage may support a view that an objective fat percentage measure for the LD could be an effective substitute for visual assessment. If confirmed by more extensive study, including sensory evaluation, to evaluate potential distribution, coarseness and fineness influences within a total fat percentage, this could prioritise development of non invasive fat measurement in the hot or cold carcase. To summarise, we have found that the image analysis is evaluating marbling in a significantly different way to grader assessment and NIR measures. Results of the study will assist in development of an eating quality assessment system for Polish beef and provides evidence to help inform any improvements to existing international systems.

\section{Conclusion}

It was concluded that EUROP carcase measures were not useful in predicting marbling of cuts whereas USDA and MSA marbling scores from independent experienced assessors were highly correlated, between the two systems and between carcase muscles although the strength of this relationship varied by muscle with oyster blade (M.infraspinatis) an 
exception. NIR values were also well correlated with the USDA and MSA values from which it was concluded that an effective on line intramuscular fat measure may potentially predict equivalent marbling. The image analysis measures were less correlated with visual grading and NIR indicating that they are evaluating marbling in a significantly different way. Individual image analysis fineness and coarseness measures were highly differentiated. It was concluded that further evaluation of measures in conjunction with sensory values would be valuable in determining the merit of detailed image analysis to quantify marbling particle fineness in addition to or in conjunction with visual grading scores.

\section{Acknowledgements}

The generous expert assistance of Dr Kari Spivey and Janine Lau in providing USDA and MSA marbling image assessment is very much appreciated. Financial support and provision of samples from the project "Optimization of beef production in Poland in accordance with the strategy from the fork to the farm" (UDA-POIG.01.03.01-00-204/09-03) is gratefully acknowledged as is the advice of Dr Ray Watson and Dr John Thompson in regard to analysis and manuscript editing. The authors would also like to thank two anonymous referees and the associate editor for their helpful comments on an earlier draft.

\section{References}

Anonymous (1996). Standards for grades of slaughter cattle and standards for grades of carcass beef. Agricultural Marketing Services, USDA. Government Printing Office, Washington, DC.

Anonymous (2005). Handbook of Australian Meat. International red meat manual (7th edn). AUS-MEAT Limited (0957879369).

Anonymous (2014). Beef Carcass Grading Standard. Japan Meat Grading Association, Tokyo, Japan.

Calkins, C., Hodgen, J. (2007). A fresh look at meat flavor. Meat Science, 77(1), 63-80.

Chen, K., Sun, X., Qin, Ch., Tang, X. (2010). Color grading of beef fat by using computer vision and support vector machine. Computers and Electronics in Agriculture, 70, 27-32. 
Ferguson, D., Thompson, J., Polkinghorne, R. (1999). Meat Standards Australia, a "PACCP" based beef grading scheme for consumers. 3) PACCP requirements which apply to carcass processing. Book of Abstracts of the 45th International Congress of Meat Science and Technology, Yokohama, Japan, 18-19.

Konarska, M., Kuchida, K., Guzek, D., Głąbska, D., Wierzbicka, A., Gutkowska, K., Polkinghorne, R. (2013). Evaluation of marbling and color of frozen beef using image analysis. 59th International Congress of Meat Science and Technology: 18-23rd August, Izmir, Turkey, 4.

Kuchida, K., Konishi, K., Suzuki, M., Miyoshi, S. (1998). Prediction of the crude fat contents in rib-eye muscle of beef using the fat area ratio calculated by computer image analysis. Nihon Chikusan Gakkaiho (Animal Science Journal), 69(6), 655-658.

Kuchida, K., Kono, S., Konishi, K., Van Vleck, L. D., Suzuki, M., Miyoshi, S. (2000). Prediction of crude fat content of longissimus muscle of beef using the ratio of fat area calculated from computer image analysis: comparison of regression equations for prediction using different input devices at different stations. Journal of Animal Science, 78(4), 799-803.

Kuchida, K., Kurihara, T., Suzuki, M., Miyoshi, S. (1997). Development of an accurate method for measuring fat percentage on rib-eye area by computer image analysis. Animal Science and Technology, 68(9), 853-859.

Kuchida, K., Osawa, T., Hori, T., Kotaka, H., Maruyama, S. (2006). Evaluation and genetics of carcass cross section of beef carcass by computer image analysis. The Journal of Animal Genetics (Japanese), 34(2), 45-52.

Kuchida, K., Suzuki, M., Miyoshi, S. (2001). Development of photographing equipment for the cross section of carcass and prediction of BMS number by using obtained image from that equipment. Nihon Chikusan Gakkaiho (Animal Science Journal), 72, 224-231.

Moore, C., Bass, P., Green, M., Chapman, P., Connor, M., Yates, L., Scanga, J., Tatum, J., Smith, G., Belk, K. (2010). Establishing an appropriate mode of comparison for measuring the performance of marbling score output from video image analysis beef carcass grading systems. Journal of Animal Science, 88, 2464-2475. 
Platter, WJ., Tatum, JD., Belk, KE., Chapman, PL., Scanga, JA., Smith, GC. (2003). Relationships of consumer sensory ratings, marbling score, and shear force value to consumer acceptance of beef strip loin steaks. Journal of Animal Science, 81, 2741-2750.

Polkinghorne, R., Thompson, J., Watson, R., Gee, A., Porter, M. (2008). Evolution of the Meat Standards Australia (MSA) beef grading system. Australian Journal of Experimental Agriculture, 48(11), 1351-1359.

Prevolnik, M., Čandek-Potokar, M., \& Škorjanc, D. (2004). Ability of NIR spectroscopy to predict meat chemical composition and quality - a review. Czech Journal of Animal Science, 49(11), 500-510.

R Core Team (2016). R: A language and environment for statistical computing. R Foundation for Statistical Computing, Vienna, Austria. URL https://www.R-project.org/

Smith, D., Griffin, D., Johnson, K. (2013). Meat Evaluation Handbook (second edition). American Meat Science Association. Champaign, IL, USA.

Smith, G., Tatum, J., Belk, K., Scanga, J. (2005). Beef carcass quality and yield grading in the USA, Canada and Australia. II Jornada Tecnica de la Fundación ANETIF, Mexico City, 1314 June 2005, 1-8.

Tapp, W., Yancey, J., Apple, J. (2011). How is the instrumental color of meat measured? Meat Science, 89, 1-5.

Thompson, J. (2002). Managing meat tenderness. Meat Science, 62(3), 295-308.

Watson, R., Polkinghorne, R., Thompson, J. (2008) Development of the Meat Standards Australia (MSA) prediction model for beef palatability. Australian Journal of Experimental Agriculture, 48, 1368-1379.

Wickham, H. (2009). ggplot2: Elegant Graphics for Data Analysis. Springer-Verlag, New York.

Wood, J., Richardson, R., Nute, G., Fisher, A., Campo, M., Kasapidou, E., Sheard, P., Enser, M. (2004). Effects of fatty acids on meat quality: a review. Meat science, 66(1), 21-32. 

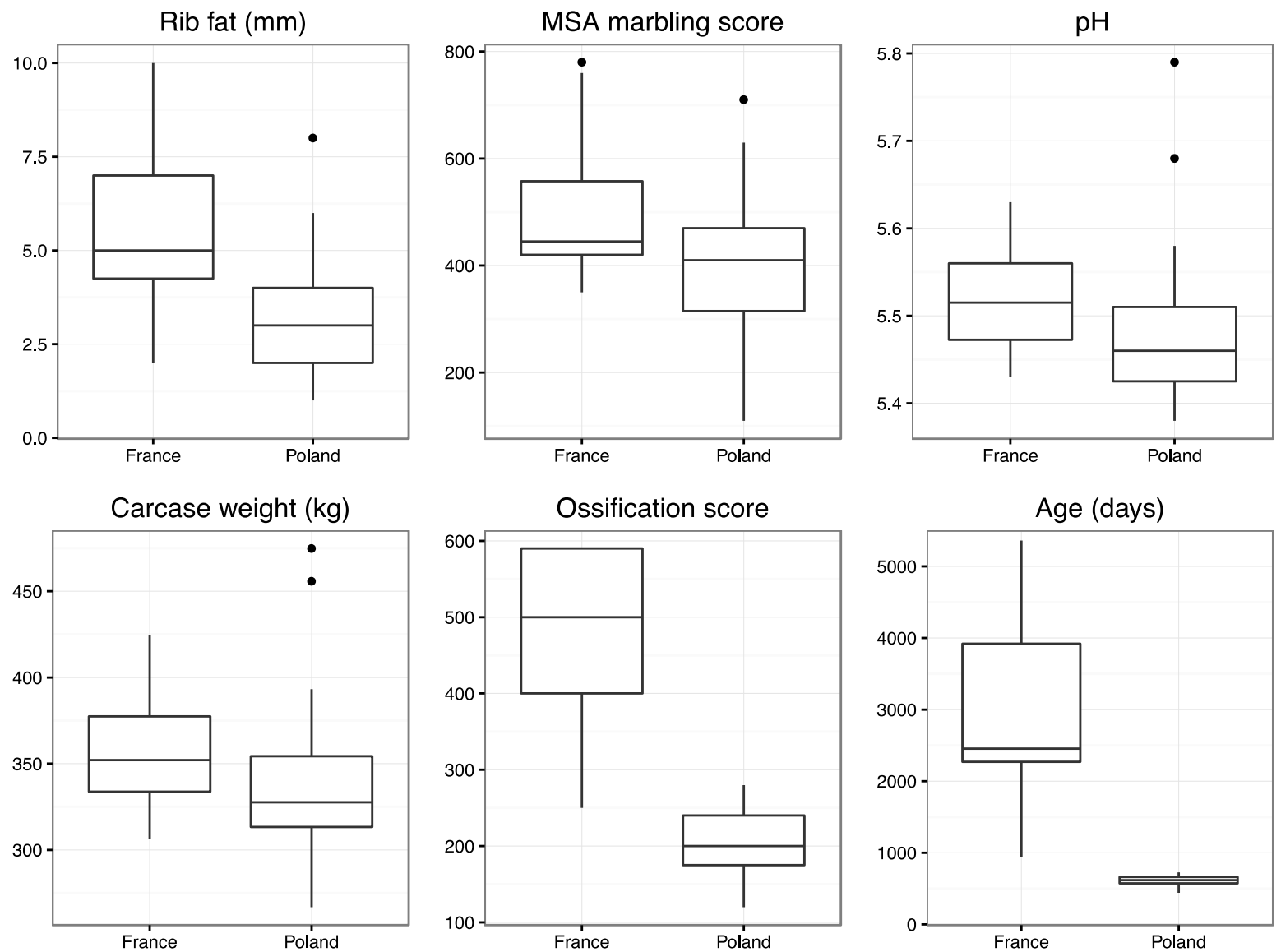

Fig. 1. Comparison of French and Polish carcase measures for source carcases. ${ }^{3}$

\footnotetext{
${ }^{3}$ MSA marbling score is assessed in increments of 10 ranging from 100 to 1100 (higher scores correspond to more marbling). Ossification scores are a measure of physiological age based on the progression of cartilage turning to bone in the spinous processes along the backbone. It is assigned in increments of 10 with 100 being the lowest and 590 the highest score.
} 

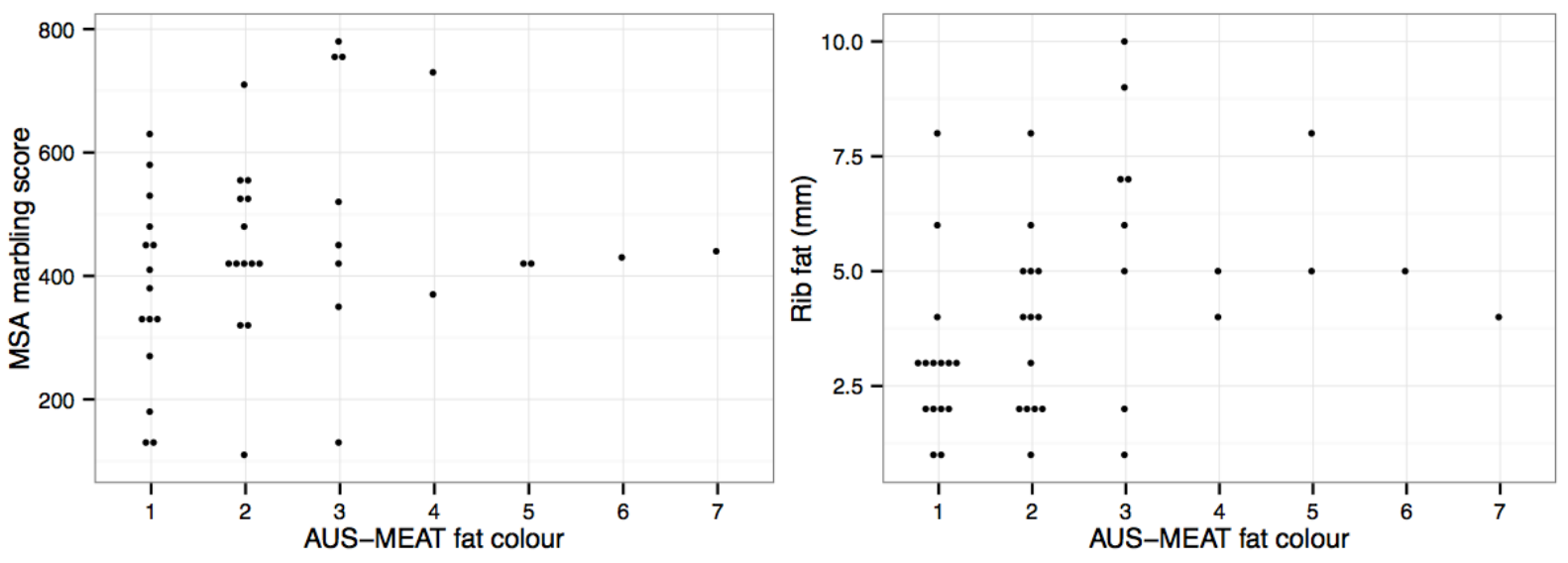

Fig. 2. Scatter plots of MSA marbling score and rib fat depth (mm) against AUS-MEAT fat colour. Overlapping observations have been stacked horizontally. 

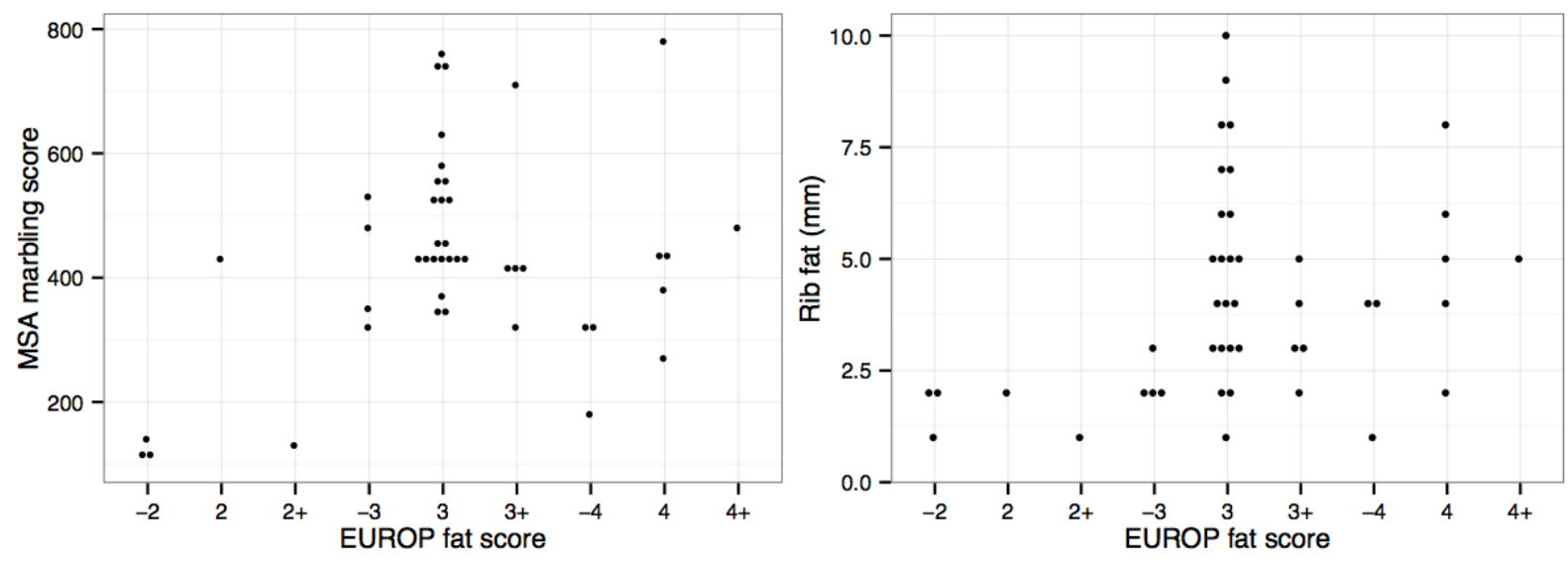

Fig. 3. Scatter plots of MSA marbling score and rib fat depth (mm) against EUROP fat score. Overlapping observations have been stacked horizontally. 


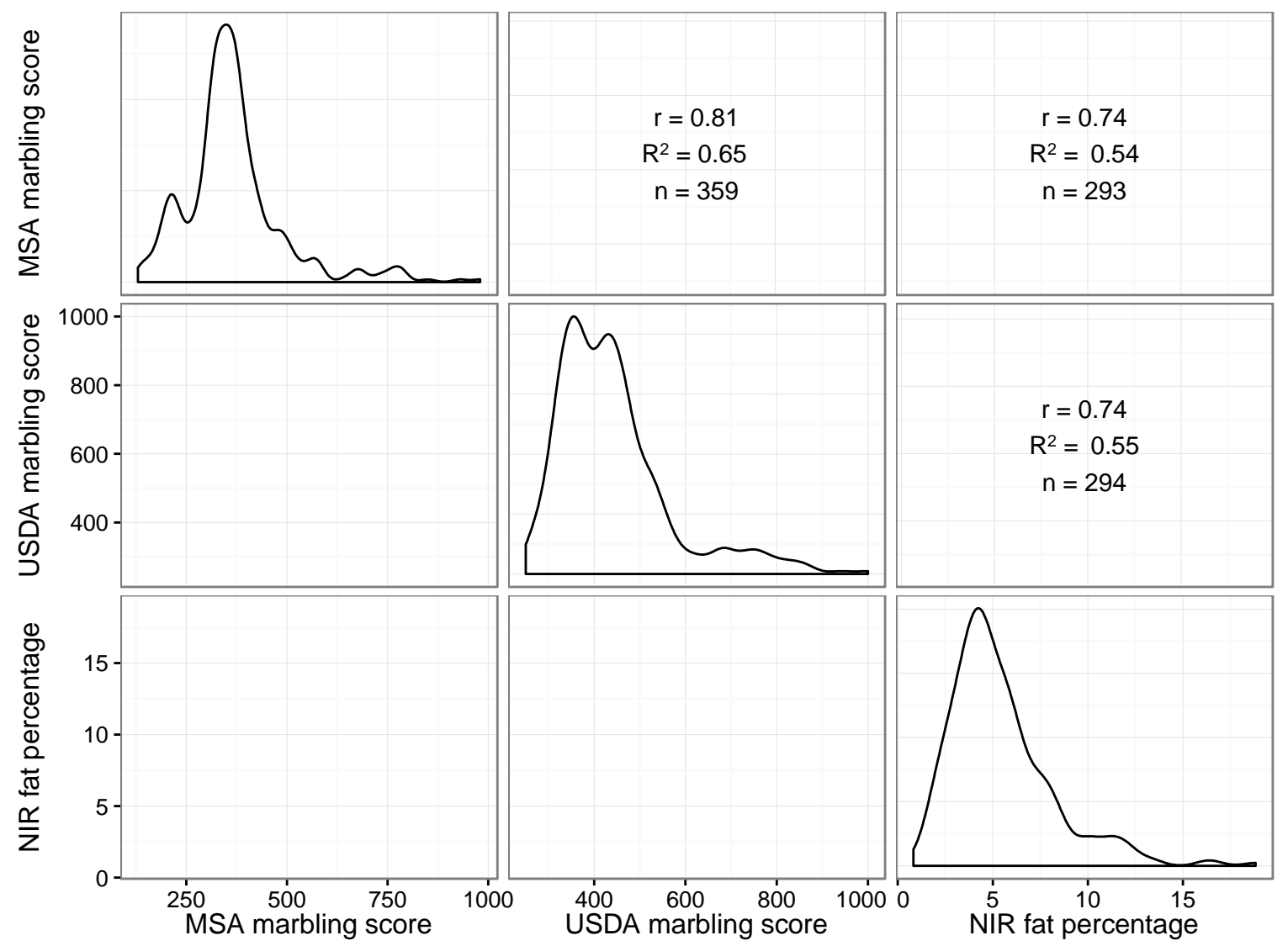

Fig. 4. Scatter plots, densities and correlations for the MSA marbling scores, USDA marbling scores and near infrared spectrophotometry (NIR) fat percentages. 


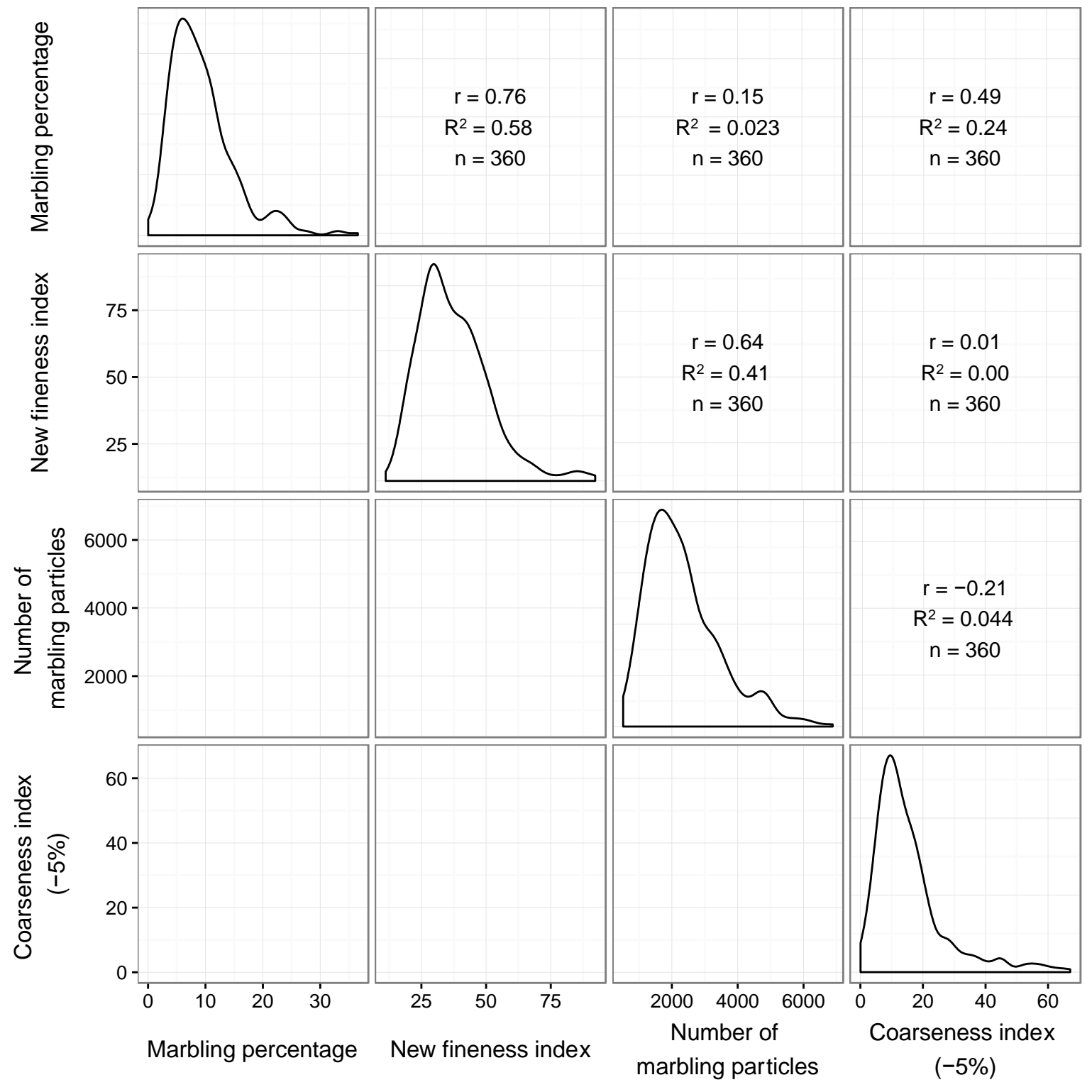

Fig. 5. Densities, scatter plots and correlations for a subset of the image analysis variables. 


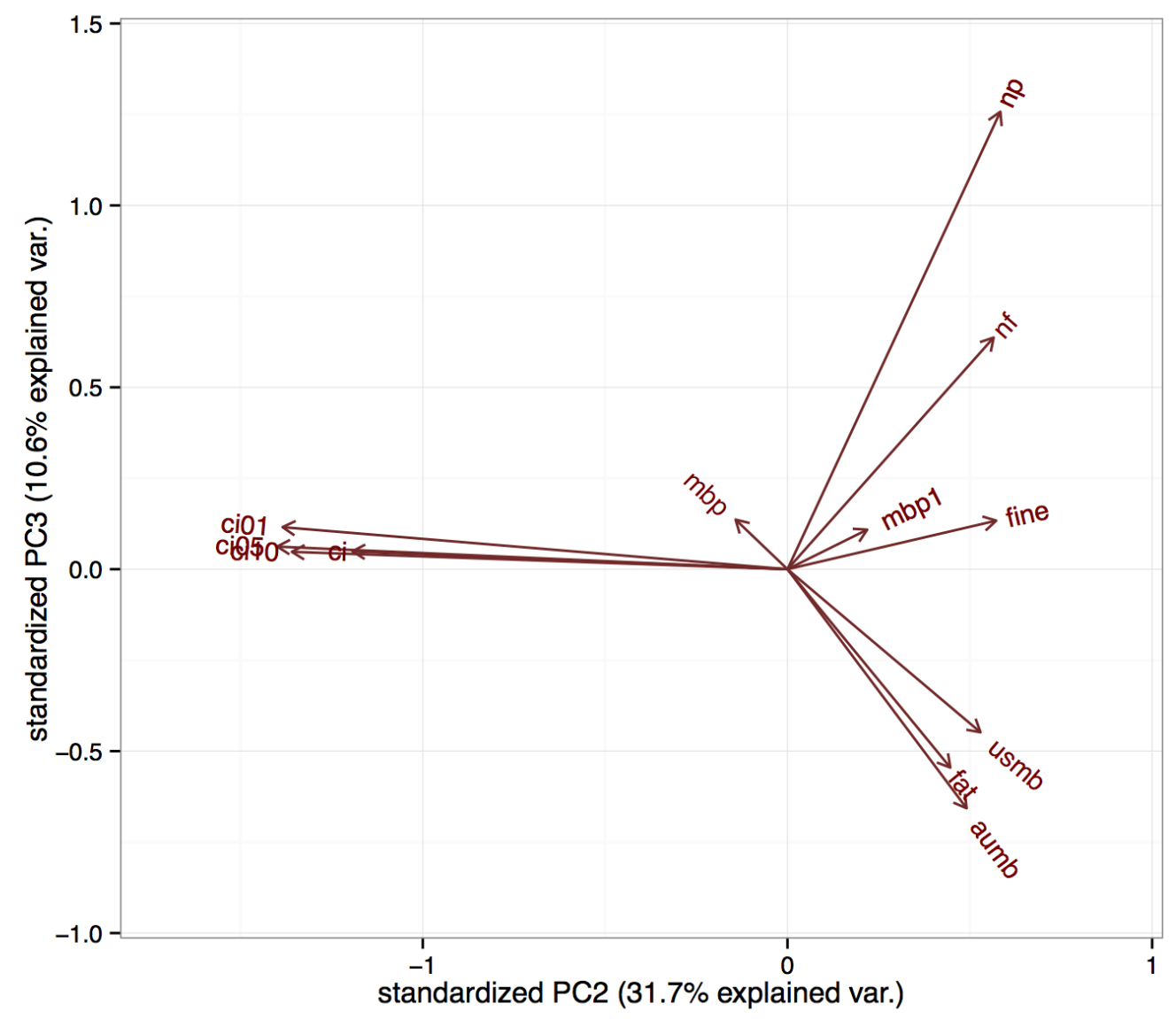

Fig. 6. Loadings of each variable on the second and third principal components. Most of the variable codes are given in Table 4. In addition, fat $=$ NIR fat percentage, usmb $=$ USDA marbling, and aumb $=$ MSA marbling . 

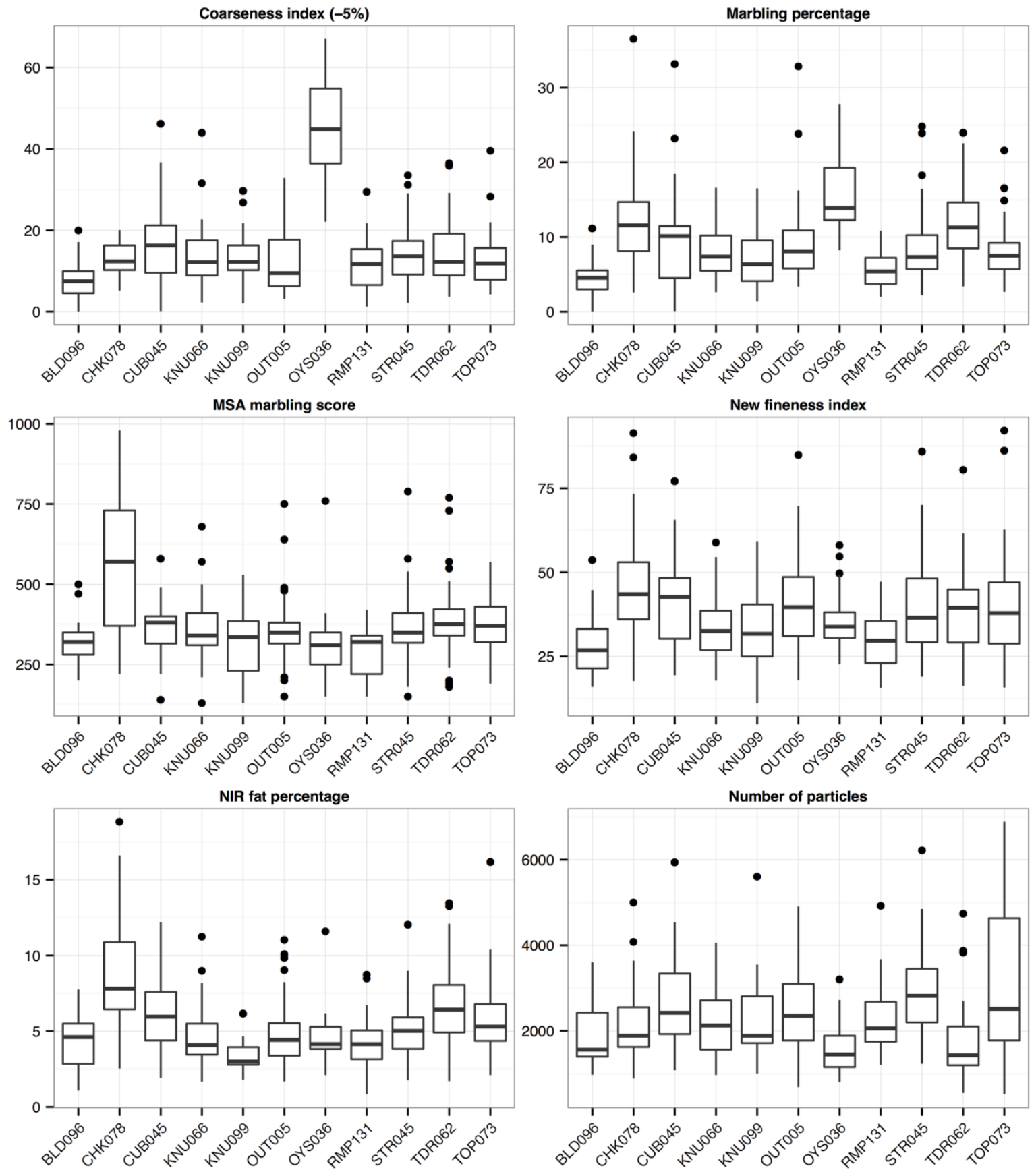

Fig. 7. Distribution of selected image analysis marbling measures by cut. MSA marbling score is assessed in increments of 10 ranging from 100 to 1100 (higher scores correspond to more marbling). ${ }^{4}$

${ }^{4}$ M. triceps brachii caput longum (BLD096); M. semispinalis capitis (CHK074); M. serratus ventralis cervicis (CHK078); M. longissimus thoracis (CUB045); M. rectus femoris (KNU066); M. vastus lateralis (KNU099); M. biceps femoris (OUT005); M. infraspinatus (OYS036); M. gluteus medius (RMP131); M. longissimus lumborum (STR045); M. psoas major (TDR062); M. semimembranosus (TOP073) 

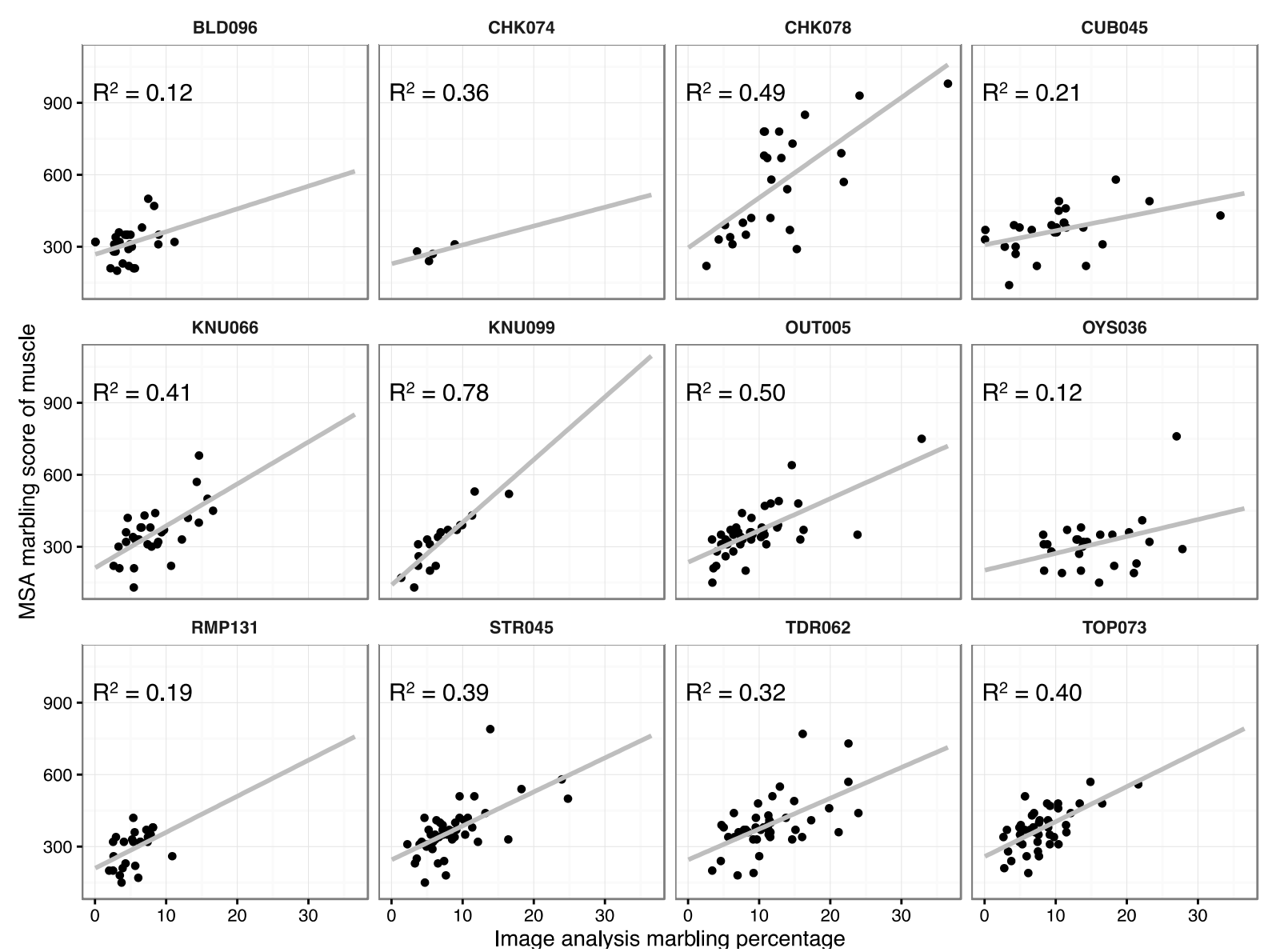

Fig. 8. MSA marbling against image analysis marbling percentage. The Pearson correlations are in the top left and the grey line is the estimated simple linear regression line. ${ }^{5}$

${ }^{5}$ M. triceps brachii caput longum (BLD096); M. semispinalis capitis (CHK074); M. serratus ventralis cervicis (CHK078); M. longissimus thoracis (CUB045); M. rectus femoris (KNU066); M. vastus lateralis (KNU099); M. biceps femoris (OUT005); M. infraspinatus (OYS036); M. gluteus medius (RMP131); M. longissimus lumborum (STR045); M. psoas major (TDR062); M. semimembranosus (TOP073) 


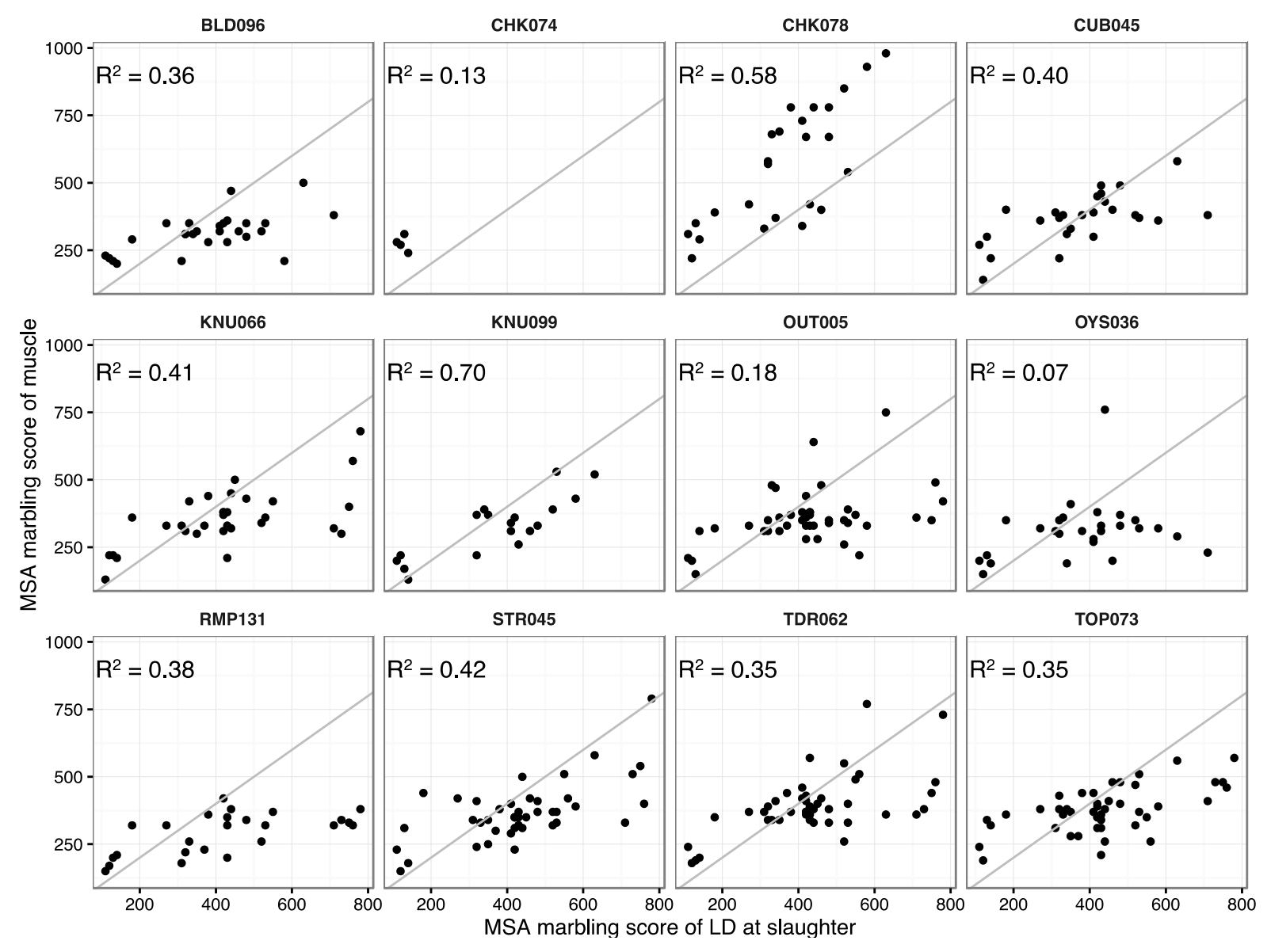

Fig. 9. Scatterplots of the MSA marbling score from photographic assessment of thawed samples against the MSA marbling score of the carcase LTL muscle at time of grading post slaughter. The grey line is the $45^{\circ}$ line which would indicate a perfect match and Pearson correlations are given in the top left of each plot. ${ }^{6}$

${ }^{6}$ M. triceps brachii caput longum (BLD096); M. semispinalis capitis (CHK074); M. serratus ventralis cervicis (CHK078); M. longissimus thoracis (CUB045); M. rectus femoris (KNU066); M. vastus lateralis (KNU099); M. biceps femoris (OUT005); M. infraspinatus (OYS036); M. gluteus medius (RMP131); M. longissimus lumborum (STR045); M. psoas major (TDR062); M. semimembranosus (TOP073) 


\section{Table 1}

Sample numbers examined by image analysis by cut and country of origin.

\begin{tabular}{|c|c|c|c|c|}
\hline Muscle & Code & France & Poland & Total \\
\hline M. triceps brachii caput longum & BLD096 & 0 & 27 & 27 \\
\hline M. semispinalis capitis & CHK074 & 0 & 4 & 4 \\
\hline M. serratus ventralis cervicis & CHK078 & 0 & 25 & 25 \\
\hline M. longissimus thoracis & CUB045 & 0 & 26 & 26 \\
\hline M. rectus femoris & KNU066 & 17 & 14 & 31 \\
\hline M. vastus lateralis & KNU099 & 0 & 18 & 18 \\
\hline M. biceps femoris & OUT005 & 16 & 27 & 43 \\
\hline M. infraspinatus & OYS036 & 0 & 27 & 27 \\
\hline M. gluteus medius & RMP131 & 11 & 14 & 25 \\
\hline M. longissimus lumborum & STR045 & 18 & 26 & 44 \\
\hline M. psoas major & TDR062 & 18 & 27 & 45 \\
\hline \multirow[t]{2}{*}{ M. semimembranosus } & TOP073 & 18 & 27 & 45 \\
\hline & Total & 98 & 262 & 360 \\
\hline
\end{tabular}


Table 2

Carcase counts for categorical variables.

\begin{tabular}{|c|c|c|c|c|c|c|c|c|c|c|c|}
\hline EUROP fatness ${ }^{1}$ & -2 & 2 & $2+$ & -3 & 3 & $3+$ & -4 & 4 & $4+$ & & \\
\hline France & - & - & - & - & 17 & - & - & 1 & - & & \\
\hline Poland & 3 & 1 & 1 & 4 & 5 & 5 & 3 & 4 & 1 & & \\
\hline $\begin{array}{l}\text { EUROP } \\
\text { conformation }^{2}\end{array}$ & E- & $\mathbf{U}+$ & $\mathbf{U}$ & U- & $\mathbf{R}+$ & $\mathbf{R}$ & R- & O+ & $\mathbf{O}$ & O- & $\mathbf{P +}$ \\
\hline France & - & - & - & - & 1 & 5 & 5 & 1 & 1 & 3 & 2 \\
\hline Poland & 3 & 1 & 3 & 2 & 4 & 1 & - & 1 & 10 & 2 & - \\
\hline $\begin{array}{l}\text { AUS-MEAT } \\
\text { fat colour }^{3}\end{array}$ & 1 & 2 & 3 & 4 & 5 & 6 & 7 & & & & \\
\hline France & - & 5 & 7 & 2 & 2 & 1 & 1 & & & & \\
\hline Poland & 15 & 10 & 1 & - & - & - & - & & & & \\
\hline $\begin{array}{l}\text { AUS-MEAT } \\
\text { meat colour }^{4}\end{array}$ & $1 \mathrm{~A}$ & $1 B$ & $1 \mathrm{C}$ & 2 & 3 & 4 & 5 & & & & \\
\hline France & - & - & 2 & 3 & 3 & 9 & 1 & & & & \\
\hline Poland & 2 & 4 & 11 & 5 & 1 & 2 & 2 & & & & \\
\hline
\end{tabular}

${ }^{1}$ The EUROP system utilises 5 fat classes from 1 to 5 with 1 the leanest and 5 fattest. These five classes can be further subdivided into - or + designations to provide a 15 point scale.

${ }^{2}$ EUROP conformation scores are designated from E (highest muscularity) to P (poorest) and can also be subdivided into + or - to create a 15 point description.

${ }^{3}$ AUS-MEAT fat colour scores relate to 8 standard colour chips used in grading. The range is from 0 (most white) to 9 (most yellow) with 9 being any measure beyond the 8 chip.

${ }^{4}$ AUS-MEAT meat colour scores relate to standard colour chips used in grading. The standards commence at $1 \mathrm{a}, 1 \mathrm{~b}$ and $1 \mathrm{c}$ then continue as whole numbers from 2 to 7 with 7 being anything darker than the 6 chip. 


\section{Table 3}

Pearson correlations of image analysis measures $(n=360) .^{7}$

\begin{tabular}{|c|c|c|c|c|c|c|c|c|c|c|}
\hline & & np & mbp & mbp1 & fine & nf & ci & ci10 & ci05 & ci01 \\
\hline $\begin{array}{l}\text { Number of } \\
\text { marbling particles }\end{array}$ & $\mathbf{n p}$ & 1 & & & & & & & & \\
\hline Marbling percentage & mbp & $0.15^{*}$ & 1 & & & & & & & \\
\hline $\begin{array}{l}\text { Marbling percentage } \\
\text { without largest particle }\end{array}$ & mbp1 & $0.23 *$ & $0.95 *$ & 1 & & & & & & \\
\hline Fineness index & fine & $0.33 *$ & $0.79 *$ & $0.89 *$ & 1 & & & & & \\
\hline New fineness index & nf & $0.64 *$ & $0.76^{*}$ & $0.85^{*}$ & $0.90 *$ & 1 & & & & \\
\hline Coarseness index & ci & $-0.13^{*}$ & $0.66^{*}$ & $0.46^{*}$ & $0.18 *$ & $0.21 *$ & 1 & & & \\
\hline Coarseness index $(-10 \%)$ & ci10 & $-0.20 *$ & $0.53 *$ & $0.30 *$ & 0.03 & 0.05 & $0.98 *$ & 1 & & \\
\hline Coarseness index $(-5 \%)$ & ci05 & $-0.21 *$ & $0.49 *$ & $0.24 *$ & -0.02 & 0.01 & $0.95 *$ & $0.99 *$ & 1 & \\
\hline Coarseness index $(-1 \%)$ & ci01 & $-0.20 *$ & $0.40^{*}$ & 0.11 & -0.07 & -0.04 & $0.85 *$ & $0.91 *$ & $0.94 *$ & 1 \\
\hline
\end{tabular}

7 The presence of a $*$ indicates that the correlation is significantly different to zero at the $1 \%$ level of significance. 


\section{Table 4}

Pearson correlations between selected image analysis variables, observed carcase marbling and NIR ${ }^{8}$.

\begin{tabular}{lccc}
\hline Image analysis variables & $\begin{array}{c}\text { MSA marbling } \\
(\mathrm{n}=359)\end{array}$ & $\begin{array}{c}\text { USDA marbling } \\
(\mathrm{n}=360)\end{array}$ & $\begin{array}{c}\text { NIR fat percentage } \\
(\mathrm{n}=294)\end{array}$ \\
\hline Number of particles & 0.03 & $0.15^{*}$ & 0.06 \\
Marbling percentage & $0.55^{*}$ & $0.53^{*}$ & $0.60^{*}$ \\
Marbling percentage without largest particle & $0.64^{*}$ & $0.62^{*}$ & $0.65^{*}$ \\
Coarseness index & $0.16^{*}$ & $0.14^{*}$ & $0.20^{*}$ \\
Coarseness index (-10\%) & 0.02 & 0.00 & 0.07 \\
Coarseness index (-5\%) & -0.03 & -0.04 & 0.02 \\
Coarseness index (-1\%) & -0.10 & -0.10 & -0.06 \\
Fineness index & $0.70^{*}$ & $0.69^{*}$ & $0.66^{*}$ \\
New fineness index & $0.55^{*}$ & $0.61^{*}$ & $0.54^{*}$ \\
\hline
\end{tabular}

8 The presence of a $*$ indicates that the correlation is significantly different to zero at the $1 \%$ level of significance. 
Table 5

Correlations between MSA marbling from photographic assessment and alternative marbling measures by cut derived from image analysis. ${ }^{9}$

\begin{tabular}{|c|c|c|c|c|c|c|}
\hline Muscle code & $\mathbf{n}$ & $\begin{array}{c}\text { NIR fat } \\
\text { percentage }\end{array}$ & $\begin{array}{l}\text { Marbling } \\
\text { percentage }\end{array}$ & $\begin{array}{l}\text { Number of } \\
\text { particles }\end{array}$ & $\begin{array}{l}\text { Coarseness } \\
\text { index }(-5 \%)\end{array}$ & $\begin{array}{l}\text { New fineness } \\
\text { index }\end{array}$ \\
\hline BLD096 & 27 & $0.80^{*}$ & 0.34 & -0.16 & 0.28 & 0.21 \\
\hline СНК078 & 25 & $0.90^{*}$ & $0.70^{*}$ & 0.02 & 0.27 & $0.59^{*}$ \\
\hline CUB045 & 26 & $0.75^{*}$ & 0.46 & -0.28 & 0.24 & 0.24 \\
\hline KNU066 & 31 & $0.91^{*}$ & $0.64 *$ & -0.09 & 0.00 & $0.56^{*}$ \\
\hline KNU099 & 18 & $0.73^{*}$ & $0.89^{*}$ & -0.09 & 0.25 & $0.68^{*}$ \\
\hline OUT005 & 43 & $0.62^{*}$ & $0.71^{*}$ & 0.06 & 0.25 & $0.64 *$ \\
\hline OYS036 & 27 & $0.86^{*}$ & 0.35 & -0.14 & -0.25 & 0.38 \\
\hline RMP131 & 25 & $0.60^{*}$ & 0.44 & 0.08 & -0.03 & 0.45 \\
\hline STR045 & 44 & $0.84 *$ & $0.63^{*}$ & 0.08 & 0.09 & $0.50^{*}$ \\
\hline TDR062 & 45 & 0.41 & $0.56^{*}$ & 0.09 & -0.02 & $0.54 *$ \\
\hline TOP073 & 45 & $0.64 *$ & $0.64 *$ & 0.17 & 0.06 & $0.51^{*}$ \\
\hline
\end{tabular}

9 The presence of a $*$ indicates that the correlation is significantly different to zero at the $1 \%$ level of significance. M. triceps brachii caput longum (BLD096); M. semispinalis capitis (CHK074); M. serratus ventralis cervicis (CHK078); M. longissimus thoracis (CUB045); M. rectus femoris (KNU066); M. vastus lateralis (KNU099); M. biceps femoris (OUT005); M. infraspinatus (OYS036); M. gluteus medius (RMP131); M. longissimus lumborum (STR045); M. psoas major (TDR062); M. semimembranosus (TOP073) 


\section{Table 6}

Correlations between NIR fat percentage and image analysis output by muscle within carcase. ${ }^{10}$

\begin{tabular}{|c|c|c|c|c|c|}
\hline Muscle code & $\mathbf{n}$ & $\begin{array}{c}\text { Marbling } \\
\text { percentage }\end{array}$ & $\begin{array}{c}\text { Number of } \\
\text { particles }\end{array}$ & $\begin{array}{c}\text { Coarseness index } \\
\qquad(-5 \%)\end{array}$ & $\begin{array}{c}\text { New fineness } \\
\text { index }\end{array}$ \\
\hline BLD096 & 21 & 0.43 & 0.01 & 0.42 & 0.38 \\
\hline CHK078 & 15 & $0.77 *$ & -0.14 & 0.43 & 0.59 \\
\hline CUB045 & 21 & 0.57 & -0.03 & 0.14 & 0.51 \\
\hline KNU066 & 28 & $0.71 *$ & 0.13 & 0.02 & $0.72 *$ \\
\hline KNU099 & 16 & 0.62 & -0.06 & -0.02 & 0.48 \\
\hline OUT005 & 37 & $0.58 *$ & -0.07 & 0.13 & $0.48 *$ \\
\hline OYS036 & 16 & 0.56 & 0.17 & -0.23 & $0.69 *$ \\
\hline RMP131 & 24 & 0.13 & 0.19 & -0.18 & 0.22 \\
\hline STR045 & 34 & $0.59 *$ & 0.14 & 0.02 & 0.47 \\
\hline TDR062 & 39 & $0.48 *$ & 0.27 & 0.04 & $0.50 *$ \\
\hline TOP073 & 40 & $0.64 *$ & 0.15 & 0.08 & $0.47 *$ \\
\hline
\end{tabular}

10 The presence of a * indicates that the correlation is significantly different to zero at the $1 \%$ level of significance. M. triceps brachii caput longum (BLD096); M. semispinalis capitis (CHK074); M. serratus ventralis cervicis (CHK078); M. longissimus thoracis (CUB045); M. rectus femoris (KNU066); M. vastus lateralis (KNU099); M. biceps femoris (OUT005); M. infraspinatus (OYS036); M. gluteus medius (RMP131); M. longissimus lumborum (STR045); M. psoas major (TDR062); M. semimembranosus (TOP073) 


\section{Table 7}

Correlations between image analysis marbling percentage and other image analysis variables. ${ }^{11}$

\begin{tabular}{lcccc}
\hline Muscle code & n & Number of particles & $\begin{array}{c}\text { Coarseness index } \\
\mathbf{( - 5 \% )}\end{array}$ & New fineness index \\
\hline BLD096 & 27 & 0.45 & 0.48 & $0.70^{*}$ \\
CHK078 & 25 & 0.33 & 0.29 & $0.90^{*}$ \\
CUB045 & 26 & 0.19 & $0.59^{*}$ & $0.71^{*}$ \\
KNU066 & 31 & 0.29 & 0.28 & $0.89^{*}$ \\
KNU099 & 18 & 0.16 & 0.33 & $0.87^{*}$ \\
OUT005 & 43 & 0.15 & 0.36 & $0.88^{*}$ \\
OYS036 & 27 & 0.24 & 0.40 & $0.63^{*}$ \\
RMP131 & 25 & 0.32 & 0.28 & $0.89^{*}$ \\
STR045 & 44 & $0.46^{*}$ & 0.26 & $0.89^{*}$ \\
TDR062 & 45 & $0.42^{*}$ & $0.50^{*}$ & \\
\hline
\end{tabular}

11 The presence of $\mathrm{a} *$ indicates that the correlation is significantly different to zero at the $1 \%$ level of significance. M. triceps brachii caput longum (BLD096); M. semispinalis capitis (CHK074); M. serratus ventralis cervicis (CHK078); M. longissimus thoracis (CUB045); M. rectus femoris (KNU066); M. vastus lateralis (KNU099); M. biceps femoris (OUT005); M. infraspinatus (OYS036); M. gluteus medius (RMP131); M. longissimus lumborum (STR045); M. psoas major (TDR062); M. semimembranosus (TOP073) 


\section{Table 8}

Correlations between MSA LTL marbling assessed at grading and alternative marbling measures by cut derived by image analysis. ${ }^{12}$

\begin{tabular}{lccccccc}
\hline $\begin{array}{l}\text { Muscle } \\
\text { code }\end{array}$ & $\mathbf{n}$ & $\begin{array}{c}\text { MSA } \\
\text { marbling }\end{array}$ & $\begin{array}{c}\text { NIR fat } \\
\text { percentage }\end{array}$ & $\begin{array}{c}\text { Marbling } \\
\text { percentage }\end{array}$ & $\begin{array}{c}\text { Number of } \\
\text { particles }\end{array}$ & $\begin{array}{c}\text { Coarseness } \\
\text { index (-5\%) }\end{array}$ & $\begin{array}{c}\text { New } \\
\text { fineness } \\
\text { index }\end{array}$ \\
\hline BLD096 & 27 & $0.60^{*}$ & $0.60^{*}$ & 0.26 & -0.10 & 0.04 & 0.15 \\
CHK078 & 25 & $0.76^{*}$ & $0.81^{*}$ & $0.57^{*}$ & -0.07 & 0.26 & 0.44 \\
CUB045 & 26 & $0.63^{*}$ & $0.65^{*}$ & 0.22 & -0.15 & 0.02 & 0.16 \\
KNU066 & 31 & $0.64^{*}$ & $0.64^{*}$ & 0.34 & -0.15 & -0.19 & 0.31 \\
KNU099 & 18 & $0.84^{*}$ & $0.71^{*}$ & $0.69^{*}$ & -0.29 & 0.36 & 0.46 \\
OUT005 & 43 & $0.42^{*}$ & $0.63^{*}$ & $0.43^{*}$ & -0.22 & 0.04 & 0.35 \\
OYS036 & 27 & 0.26 & 0.16 & 0.37 & -0.31 & 0.14 & 0.06 \\
RMP131 & 25 & $0.61 *$ & $0.62^{*}$ & 0.17 & -0.04 & -0.04 & 0.19 \\
STR045 & 44 & $0.65^{*}$ & $0.72^{*}$ & 0.33 & 0.04 & -0.16 & 0.31 \\
TDR062 & 45 & $0.60^{*}$ & $0.65^{*}$ & $0.43^{*}$ & 0.21 & -0.10 & $0.45^{*}$ \\
TOP073 & 45 & $0.59^{*}$ & $0.61 *$ & $0.58^{*}$ & 0.38 & 0.10 & $0.54 *$ \\
\hline
\end{tabular}

12 The presence of a $*$ indicates that the correlation is significantly different to zero at the $1 \%$ level of significance. M. triceps brachii caput longum (BLD096); M. semispinalis capitis (CHK074); M. serratus ventralis cervicis (CHK078); M. longissimus thoracis (CUB045); M. rectus femoris (KNU066); M. vastus lateralis (KNU099); M. biceps femoris (OUT005); M. infraspinatus (OYS036); M. gluteus medius (RMP131); M. longissimus lumborum (STR045); M. psoas major (TDR062); M. semimembranosus (TOP073) 


\section{Table 9}

Correlations between STR045 MSA marbling image assessment and alternative marbling measures by cut. ${ }^{13}$

\begin{tabular}{lccccccc}
\hline $\begin{array}{l}\text { Muscle } \\
\text { code }\end{array}$ & n & $\begin{array}{c}\text { MSA } \\
\text { marbling }\end{array}$ & $\begin{array}{c}\text { NIR fat } \\
\text { percentage }\end{array}$ & $\begin{array}{c}\text { Marbling } \\
\text { percentage }\end{array}$ & $\begin{array}{c}\text { Number of } \\
\text { particles }\end{array}$ & $\begin{array}{c}\text { Coarseness } \\
\text { index (-5\%) }\end{array}$ & $\begin{array}{c}\text { New } \\
\text { fineness } \\
\text { index }\end{array}$ \\
\hline BLD096 & 26 & $0.68^{*}$ & $0.66^{*}$ & 0.42 & 0.07 & 0.20 & 0.32 \\
CHK078 & 24 & $0.62^{*}$ & $0.81^{*}$ & 0.40 & -0.30 & 0.20 & 0.20 \\
CUB045 & 25 & $0.83^{*}$ & $0.78^{*}$ & $0.51^{*}$ & -0.17 & 0.11 & 0.34 \\
KNU066 & 31 & $0.71^{*}$ & $0.73^{*}$ & 0.44 & -0.12 & 0.08 & 0.33 \\
KNU099 & 17 & $0.73^{*}$ & $0.90^{*}$ & $0.63 *$ & -0.13 & 0.25 & 0.48 \\
OUT005 & 42 & $0.49^{*}$ & $0.73^{*}$ & $0.43 *$ & -0.04 & 0.07 & 0.37 \\
OYS036 & 26 & $0.53^{*}$ & 0.63 & 0.39 & -0.20 & -0.08 & 0.26 \\
RMP131 & 25 & $0.62^{*}$ & $0.74 *$ & 0.36 & 0.15 & 0.03 & 0.38 \\
STR045 & 44 & $1.00^{*}$ & $0.84^{*}$ & $0.63 *$ & 0.08 & 0.09 & $0.50^{*}$ \\
TDR062 & 44 & $0.54^{*}$ & $0.56^{*}$ & $0.52^{*}$ & 0.10 & 0.13 & $0.41 *$ \\
\hline
\end{tabular}

13 The presence of a $*$ indicates that the correlation is significantly different to zero at the $1 \%$ level of significance. M. triceps brachii caput longum (BLD096); M. semispinalis capitis (CHK074); M. serratus ventralis cervicis (CHK078); M. longissimus thoracis (CUB045); M. rectus femoris (KNU066); M. vastus lateralis (KNU099); M. biceps femoris (OUT005); M. infraspinatus (OYS036); M. gluteus medius (RMP131); M. longissimus lumborum (STR045); M. psoas major (TDR062); M. semimembranosus (TOP073) 